\title{
Spectral analysis of the dipping LMXB system XB 1916-053
}

\author{
A. F. Gambino ${ }^{1}$, R. Iaria ${ }^{1}$, T. Di Salvo ${ }^{1}$, S. M. Mazzola ${ }^{1}$, A. Marino ${ }^{1,4,5}$, L. Burderi $^{2}$, A. Riggio ${ }^{2}$,
} A. Sanna' ${ }^{2}$, and N. D'Amico ${ }^{3}$

\author{
1 Università degli Studi di Palermo, Dipartimento di Fisica e Chimica, Via Archirafi 36, 90123 Palermo, Italy \\ e-mail: angelofrancesco.gambino@unipa.it \\ 2 Università degli Studi di Cagliari, Dipartimento di Fisica, SP Monserrato-Sestu, KM 0.7, 09042 Monserrato, Italy \\ 3 INAF-Osservatorio Astronomico di Cagliari, Via della Scienza 5, 09047 Selargius, Italy \\ ${ }^{4}$ INAF/IASF Palermo, Via Ugo La Malfa 153, 90146 Palermo, Italy \\ 5 IRAP, Université de Toulouse, CNRS, UPS, CNES, Toulouse, France
}

Received 8 March 2018 / Accepted 3 April 2019

\begin{abstract}
Context. XB 1916-053 is a low mass X-ray binary system (LMXB) hosting a neutron star (NS) and showing periodic dips. The spectrum of the persistent emission was modeled with a blackbody component having a temperature between 1.31 and $1.67 \mathrm{keV}$ and with a Comptonization component with an electron temperature of $9.4 \mathrm{keV}$ and a photon index $\Gamma$ between 2.5 and 2.9. The presence of absorption features associated with highly ionized elements suggested the presence of partially ionized plasma in the system.

Aims. In this work we performed a study of the spectrum of XB 1916-053, which aims to shed light on the nature of the seed photons that contribute to the Comptonization component.

Methods. We analyzed three Suzaku observations of XB 1916-053: the first was performed in November 2006 and the others were carried out in October 2014. We extracted the persistent spectra from each observation and combined the spectra of the most recent observations, obtaining a single spectrum with a higher statistic. We also extracted and combined the spectra of the dips observed during the same observations.

Results. On the basis of the available data statistics, we infer that the scenario in which the corona Comptonizes photons emitted both by the innermost region of the accretion disk and the NS surface is not statistically relevant with respect to the case in which only photons emitted by the NS surface are Comptonized. We find that the source is in a soft spectral state in all the analyzed observations. We detect the $\mathrm{K} \alpha$ absorption lines of Fe xxv and Fe xxvi, which have already been reported in literature, and for the first time the $\mathrm{K} \beta$ absorption lines of the same ions. We also detect an edge at $0.876 \mathrm{keV}$, which is consistent with a $\mathrm{O}$ vIII $\mathrm{K}$ absorption edge. The dip spectrum is well described by a model that considers material in different ionization states covering the persistent spectrum and absorbing part of the rear radiation. From this model we rescale the distance of the absorber to a distance that is lower than $1 \times 10^{10} \mathrm{~cm}$.
\end{abstract}

Key words. line: formation - line: identification - stars: neutron - stars: individual: XB 1916-053 - X-rays: binaries $\mathrm{X}$-rays: general

\section{Introduction}

XB 1916-053 is a low mass X-ray binary system (LMXB) showing dips and type I X-ray bursts. Type I X-ray bursts were observed for the first time by Becker et al. (1977) using OSO 8 data and revealed the presence of a neutron star (NS) in the binary system. The distance to the source was evaluated by several authors taking into account the X-ray bursts showing photospheric radius expansion (PRE), which are characterized by luminosities reaching the Eddington limit. Smale et al. (1988) evaluated a distance of $8.4 \mathrm{kpc}$ for an accreted material with cosmic abundances of hydrogen, or else of $10.8 \mathrm{kpc}$ for a hydrogendeficient material.

Galloway et al. (2008) suggested a distance of $7 \mathrm{kpc}$ or $9 \mathrm{kpc}$, for a H-rich and He-rich plasma accreted onto the NS surface, respectively. Moreover, Yoshida (1993) suggested a distance of $9.3 \mathrm{kpc}$ with a $15 \%$ error, on the basis of the study of a type I X-ray burst that showed PRE observed with the Ginga satellite.

Church et al. (1997) evaluated the orbital period of the system, taking advantage of the periodicity of the dips present in the ASCA data. The dips are evidence of the partial covering of the central emission region of the binary system by a bulge of cold and/or partially ionized material in the point at which the accretion flow hits the outer part of the accretion disk. These authors estimated an orbital period of $P=3005 \pm 10 \mathrm{~s}$. The observation in the optical band showed a modulation of the light curve with a period of $3027.4 \pm 0.4 \mathrm{~s}$ (Grindlay et al. 1988). The successive extension of the orbital ephemeris (Iaria et al. 2015) revealed that this modulation may be caused by the presence of a third body in the system. The mass of this body and its orbital period are dependent on the mass transfer scenario adopted for XB 1916-053. The authors evaluated a mass for the third body of $M_{3}=0.10-0.14 M_{\odot}$ and an orbital period of $51 \mathrm{yr}$ assuming a conservative mass transfer, or $M_{3}=0.055 M_{\odot}$ and an orbital period of $26 \mathrm{yr}$ if the mass transfer is nonconservative. In the latter case, the analysis also returned an estimation of the companion star mass $\left(M_{2}=0.028 M_{\odot}\right)$, that is a helium-rich star, according to Nelemans et al. (2006). The presence of the dips in the light curve suggests that the system is seen with a high inclination angle, i.e., between $60^{\circ}$ and $80^{\circ}$ (Frank et al. 1987).

The spectrum of XB 1916-053 has been widely investigated both for the continuum and the contribution of the dips. Zhang et al. (2014), using Suzaku data of November 2006, observed the source in a high-soft spectral state and modeled 
the continuum spectrum of XB 1916-053 taking into account a single corona that Comptonizes the photons emitted both by the NS surface and by the innermost part of the accretion disk. This model allowed these authors to constrain the spectral parameters of the accretion disk, as well as those related to the blackbody component associated with the emission of the NS surface. They obtained an equivalent hydrogen column density of $N_{\mathrm{H}}=$ $(6 \pm 1) \times 10^{20} \mathrm{~cm}^{-2}$, inferring spectral parameters that are typical of systems in a soft spectral state. They found an inner disk temperature of $k T_{\text {in }}=1.87 \pm 0.18 \mathrm{keV}$ and a temperature and photon index for the electron corona of $k T_{\mathrm{e}}=3.7_{-0.4}^{+1.8} \mathrm{keV}$ and $\Gamma=2.65_{-0.46}^{+1.28}$, respectively.

The spectrum also shows some absorption lines superimposed on the continuum emission. The doublet of the Fe xxv and Fe xxvi $\mathrm{K} \alpha$ absorption lines was detected for the first time by Boirin et al. (2004) with XMM-Newton data. The authors also found weak evidence of the presence of the $\mathrm{Mg}$ XII, S XVI, and $\mathrm{Ni}$ XxVII $\mathrm{K} \alpha$ absorption lines, as well as the possible presence of the Fe xxvI $\mathrm{K} \beta$ absorption line and an absorption edge at $0.98 \mathrm{keV}$. The authors proposed that the latter should be a $\mathrm{K}$ absorption edge from moderately ionized $\mathrm{Ne}$ ions, or an $\mathrm{L}$ absorption edge from moderately ionized Fe ions. They also proposed the possibility that this should be the result of a superimposition of the edges of both the kind of ions.

Iaria et al. (2006), using Chandra data, confirmed the presence of the Mg XII and S XVI K $\alpha$ absorption lines, and furthermore detected the $\mathrm{K} \alpha$ absorption lines of $\mathrm{Ne} \mathrm{x}$ and Si XIV. The diagnostic of the absorption lines with Chandra allowed the authors to obtain an estimation of the line widths of these narrow features and the following estimation of the emission region at which the lines originate: $4 \times 10^{10} \mathrm{~cm}$ far away from the NS, that is almost at the accretion disk outer rim.

In Boirin et al. (2005) the transition from the persistent to the dip spectrum was explained as a decrease of the ionization parameter $\xi$ of the plasma with a simultaneous increase of the equivalent hydrogen column density of a ionized absorber interposed between the observer and the central emitting source. Furthermore, because of the great variety of absorption features into the spectrum of XB 1916-053, this absorber is likely to be partially ionized plasma.

The aim of this work is the detailed analysis of the continuum and absorption lines present in the spectrum of XB 1916-053, taking advantage, for the first time, of all the available observations performed by Suzaku.

The paper is structured as follows: in Sect. 2 we report the data selection and reduction, in Sect. 3 we report the spectral analysis performed on the persistent and dip spectra, whilst we discuss the obtained results in Sect. 4. A conclusion is reported in Sect. 5.

\section{Observation and data reduction}

Suzaku (Mitsuda et al. 2007) observed XB 1916-053 three times. The first observation (ObsID 401095010; hereafter Obs 1) was performed from 8 November 2006 at 05:49:53 to 9 November 2006 at $02: 41: 15$, for a net exposure of about $39 \mathrm{ks}$. The other two observations were acquired in 2014 and in particular the first observation (ObsID 409032010, hereafter Obs 2) was performed from 14 October at 16:20:52 to 18 October at 12:20:16, for a net exposure of $155.9 \mathrm{ks}$. The second observation (ObsID 409032020; hereafter Obs3) was performed from 18 October at 12:20:17 to 22 October at 02:40:09, for a net exposure of 140.5 ks (see Fig. 1).

During all the observations both the X-ray Imaging Spectrometers (XIS; Koyama et al. 2007) and the Hard X-ray
Detector (HXD; Takahashi et al. 2007) were operative. The XIS detectors consist of four chips generally numbered from 0 to 3 that are sensible to the energy band between 0.2 and $12 \mathrm{keV}$. The XIS0, XIS2, and XIS3 have a similar response to the incoming radiation, consisting of the same kind of front-illuminated charge-coupled device (CCD). On the other hand, the XIS1 uses a back-illuminated CCD. Unfortunately, the XIS2 broke down in 2006, moreover during Obs 1, and it has shut down ever since. Therefore the only existing XIS2 data in our sample are relative to a fraction of Obs 1. The HXD assembly, on the other hand, consists of two nonimaging detectors: the p-i-n type $\mathrm{Si}$ photo diode (PIN) that is sensible to the energy band 10-70 keV and the $\mathrm{Gd}_{2} \mathrm{SiO}_{5}(\mathrm{Ce})$ scintillators (GSO) that are sensible to the energy range $30-600 \mathrm{keV}$.

In Obs 1 the XIS chips operated in full window mode and collected data edited both in $3 \times 3$ and $5 \times 5$ pixel mode for a net exposure of $40 \mathrm{ks}$, while the HXD-PIN detector was exposed for $37 \mathrm{ks}$.

On the other hand, in Obs 2, the XIS0, XIS1, and XIS3 detectors operated using the $1 / 4$ window option and collected data edited both in $3 \times 3$ and $5 \times 5$ pixel mode for a net exposure of $155.9 \mathrm{ks}$, while the HXD-PIN detector was exposed for $8.8 \mathrm{ks}$. Similar to the previous observation, in Obs 3 all the XIS detectors operated using the $1 / 4$ window option and collected data edited both in $3 \times 3$ and $5 \times 5$ pixel mode for a net exposure of $140.5 \mathrm{ks}$, whilst the HXD-PIN collected data for $6.9 \mathrm{ks}$.

The source was not detected in the GSO, and for this reason we limited our analysis to the spectra collected by the XIS and PIN detectors for all the observations. The data were analyzed inside the HEASoft v. 6.19 environment. Moreover, for Obs 1 we used the calibration files v. 20070731 and v. 20070710 for XIS and HXD/PIN, respectively. Similarly, for Obs 2 and Obs 3 we used the XIS calibration files v. 20160607 and the HXD-PIN calibration files v. 20110913.

All the data were processed with the aepipeline routine provided with the Heasoft FTOOLS. For each XIS chip we combined the data edited in $3 \times 3$ and $5 \times 5$ pixel format and performed a better estimation of the attitude using the aeattcor.sl tool created and distributed by J. E. Davis. The attitude correction to the XIS data was applied with the supplementary tool xiscoord.

The light curve of Obs 1 showed an increasing trend in the count rate from about $5 \mathrm{c} \mathrm{s}^{-1}$ up to $11 \mathrm{c} \mathrm{s}^{-1}$. The observation showed periodic dips and one type I X-ray burst, as already observed by Zhang et al. (2014).

Instead, the Obs 2 initial count rate of about $18 \mathrm{cs}^{-1}$ decreased until a final value of about $5 \mathrm{c} \mathrm{s}^{-1}$. Four type I X-ray bursts are present in the observation as well as several periodic dips. The hardness ratio of the source during the observation, however, did not show significant variations and maintained an approximately constant trend. On the other hand, during Obs 3 the source maintained an approximately constant count rate of about $5 \mathrm{c} \mathrm{s}^{-1}$ and several periodic dips are present. Also in this case, the hardness ratio did not show any important variations.

In this work we were not interested in the analysis of the type I X-ray bursts; we then removed a $5 \mathrm{~s}$ time segment before the rise time of each burst and a time interval $100 \mathrm{~s}$ long subsequent the onset of the burst. Furthermore, we distinguished between the dip and the persistent emission, performing a time selection on the data of all the observations.

To estimate if the XIS data were affected by pileup, we used the tool pile-estimate.sl created by M. A. Novak. For Obs 1 we obtained a pileup fraction between $9 \%$ and $13 \%$ for all the XIS chips considering the events in a circular region centered at the coordinates of XB 1916-053 $\left(\mathrm{RA}=289.699462^{\circ}\right.$ 


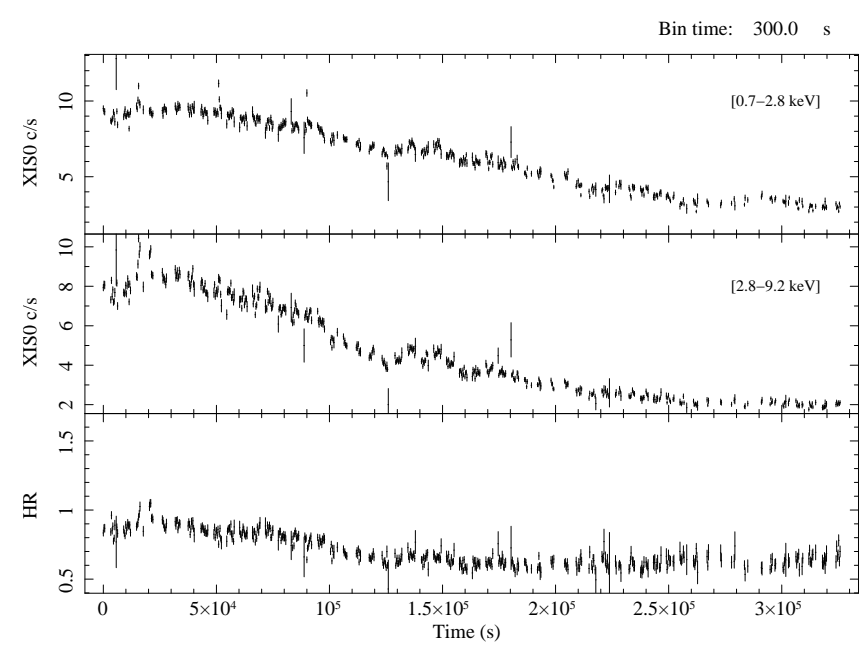

Start Time 16944 17:09:06:184 Stop Time 16948 11:39:06:184

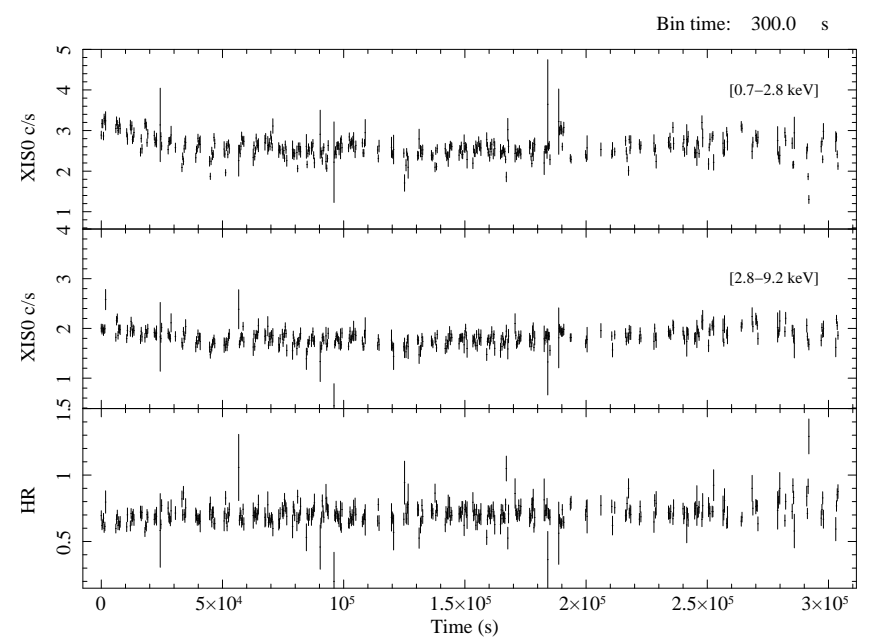

Start Time 16948 12:51:38:436 Stop Time 16952 1:21:38:436

Fig. 1. Light curves from XIS0 in the energy bands $0.7-2.8 \mathrm{keV}$ (top panels) and 2.8-9.2 keV (middle panels) for ObsID 409032010 (Obs 2 , left) and ObsID 409032020 (Obs 3, right), respectively. The corresponding hardness ratios are reported in the bottom panels.

and Dec $=-5.238081^{\circ}(\mathrm{J} 2000)$; Iaria et al. 2006) and with radius $114^{\prime \prime}$. Then, in order to reduce the pileup fraction under the $4 \%$ threshold, i.e., the tolerance limit we assumed in this work, we only considered the events included in an annular region centered onto the source and having an outer radius of $114^{\prime \prime}$ and an inner radius of $31^{\prime \prime}$. On the other hand, for Obs 2 and Obs 3 a circular region centered at the coordinates of the source and having a radius of $114^{\prime \prime}$ is sufficient to obtain an estimation of the pileup fraction of the $4 \%$ at most for Obs 2 and of about $2 \%$ for Obs 3 . These two observations, indeed, were acquired using the $1 / 4$ windows option that allows us to increase the window frame time and to observe the source without pileup also to higher count rates with respect to the full window mode. The XIS spectra of the source for each observation were obtained considering events falling inside these extraction regions. The corresponding background spectra were extracted selecting events inside a circular region localized far away from the source and covering the same area of the corresponding source extraction region. For Obs 1 we extracted the spectra of the persistent emission and of the dips taking into account the data collected by each XIS chip. For the XIS2 data, we excluded the last $6 \mathrm{ks}$ of observation during which the instrument did not acquired data anymore, owing to the damage it had undergone. As a consequence of this, we took into account only the spectra extracted from the XIS0, XIS1, and XIS3 data for Obs 2 and Obs 3.

The response of each XIS chip was obtained using the tool xisrmfgen, whilst the ancillary response files (ARFs) were obtained using the tool xissimarfgen, setting as coordinates for the source RA $=289.699462^{\circ}$ and $\mathrm{Dec}=-5.238081^{\circ}$ (Iaria et al. 2006).

The HXD-PIN spectra were extracted using the tool hxdpinxbpi, while we used the response files provided by the HXD team. We selected the response files ae_hxd_ pinxinome3_20080129.rsp for Obs 1 and ae_hxd_pinxi nome11_20110601.rsp for Obs 2 and Obs 3, respectively.

The background file of Obs 1 was regularly produced by the HXD team and then used in the analysis, whilst the background files for the Obs 2 and Obs 3 of 2014 were not available. Starting from that year, indeed, normal operation of the HXD was very rare owing to the power consumption of the spacecraft. In order to estimate the background generated by the NXB+CXB (Non-X-ray Background+Cosmic X-ray Background) events, the HXD team suggested that we use the spectrum of the source RXJ 1856.5-3754, that is a NS with a considerably soft $\mathrm{X}$-ray emission, where the PIN data are almost attributable to the $\mathrm{CXB}+\mathrm{NXB}$ contribution. Since our source is very bright with respect to RXJ 1856.5-3754, the background systematic error is not so significant below $30 \mathrm{keV}$, whilst the NXB systematics above $30 \mathrm{keV}$ may be around the 10-20\% in Obs 2 and Obs 3.

Then, we processed the ObsID 109008010 data of RXJ 1856.5-3754 of 2014 April with the aepipeline routine. This observation has an exposure of $35 \mathrm{ks}$ that has been considered in its entirety to extract the HXD/PIN spectrum of the source through the hxdpinxbpi tool. To take into account a systematic error of the $20 \%$ above $30 \mathrm{keV}$, we used the ftool grppha. Hereafter, this spectrum represents the HXD/PIN CXB + NXB background spectrum for both Obs 2 and Obs 3 .

The products of this first processing step for Obs 1 are spectra of the persistent emission of XB 1916-053 for a net exposure of $33 \mathrm{ks}$ for the XIS0 and XIS3, and $30 \mathrm{ks}$ for the XIS2. Furthermore, the corresponding HXD/PIN spectrum has an exposure of $30 \mathrm{ks}$.

From Obs 2 we extracted XIS persistent spectra of $120 \mathrm{ks}$ of exposure for each considered chip respectively and a HXD/PIN persistent spectrum of $8 \mathrm{ks}$ of exposure. Furthermore, from this observation we also extracted XIS spectra of the dips for an amount of $13 \mathrm{ks}$ of exposure for each XIS chip, as well as a HXD/PIN spectrum of the dips of $502 \mathrm{~s}$ exposure. On the other hand, for the Obs 3 we extracted XIS spectra of the persistent emission for an amount of $83 \mathrm{ks}$ of exposure for each of the considered chips, and of $1.5 \mathrm{ks}$ of exposure from the HXD/PIN data. With regard to the dip part, we extrapolated spectra of $23 \mathrm{ks}$ from the XIS data of each considered chip, as well as a HXD/PIN spectrum of $5 \mathrm{ks}$ of exposure.

Considering the similar spectral response of XIS0, XIS2, and XIS3, and once we tested the actual compatibility of the spectra acquired by these instruments, in order to increase the statistics we chose to combine the spectra extracted from these devices and their spectral responses using the addascaspec routine. We did this for all the observations and both for the persistent and the dip spectra; we remark that during the Obs 2 and Obs 3, only the XISO and XIS3 were active.

Furthermore, we noticed that the spectral shape of the persistent emission of XB 1916-053 was the same in Obs 2 and Obs 3 . Then, since these observations are extremely close in time, 
Table 1. Results of the fits on Spectrum 1 and Spectrum 2 adopting the 1-seed and 2-seed models, respectively.

\begin{tabular}{|c|c|c|c|c|c|}
\hline \multirow[b]{2}{*}{ Component } & \multirow[b]{2}{*}{ Parameter } & \multicolumn{2}{|c|}{ Model: 1-seed } & \multicolumn{2}{|c|}{ Model: 2-seed } \\
\hline & & Spectrum 1 & Spectrum 2 & Spectrum 1 & Spectrum 2 \\
\hline PHABS & $N_{\mathrm{H}}\left(10^{22}\right)$ & $0.433_{-0.010}^{+0.012}$ & $0.479_{-0.013}^{+0.014}$ & $0.45_{-0.02}^{+0.03}$ & $0.479_{-0.010}^{+0.020}$ \\
\hline \multirow[t]{3}{*}{ GABS } & LineE (keV) & - & $4.4 \pm 0.3$ & - & $4.4_{-0.3}^{+0.2}$ \\
\hline & Sigma $(\mathrm{keV})$ & - & $1.4_{-0.2}^{+0.4}$ & - & $1.4_{-0.3}^{+0.3}$ \\
\hline & Strength & - & $0.7_{-0.4}^{+1}$ & - & $0.7_{-0.2}^{+0.7}$ \\
\hline \multirow[t]{2}{*}{ EDGE } & edgeE (keV) & - & $0.876_{-0.013}^{+0.02}$ & - & $0.876_{-0.013}^{+0.014}$ \\
\hline & MaxTau & - & $0.11 \pm 0.02$ & - & $0.11 \pm 0.02$ \\
\hline \multirow[t]{3}{*}{ BBODY } & $k T(\mathrm{keV})$ & $1.3_{-0.1}^{+0.2}$ & $1.1_{-0.3}^{+0.1}$ & $1.9_{-0.4}^{+0.2}$ & $1.11_{-0.2}^{+0.03}$ \\
\hline & Norm. $\left(10^{-3}\right)$ & $<0.95$ & $1_{-1}^{+2}$ & $<1.44$ & $<3.5$ \\
\hline & вBODY UNABs. FluX $\left(\mathrm{erg} \mathrm{cm}^{2} \mathrm{~s}^{-1}\right)$ & $<8 \times 10^{-11}$ & $(1.2 \pm 0.1) \times 10^{-10}$ & $<1 \times 10^{-10}$ & $<3 \times 10^{-10}$ \\
\hline \multirow[t]{3}{*}{ DISKBB } & $T_{\text {in }}(\mathrm{keV})$ & $0.69 \pm 0.03$ & $0.55_{-0.04}^{+0.05}$ & $0.60_{-0.1}^{+0.07}$ & $0.55_{-0.04}^{+0.05}$ \\
\hline & $\left(R_{\mathrm{in}} / D_{10}\right)^{2} \cos (\theta)\left(\mathrm{km}^{2} \mathrm{kpc}^{-2}\right)$ & $44 \pm 7$ & $68_{-19}^{+25}$ & $<33$ & $68_{-19}^{+20}$ \\
\hline & DISKBb UNABS. FLuX $\left(\mathrm{erg} \mathrm{cm}^{2} \mathrm{~s}^{-1}\right)$ & $(2.0 \pm 0.2) \times 10^{-10}$ & $(1.3 \pm 0.1) \times 10^{-10}$ & $<9 \times 10^{-11}$ & $(1.3 \pm 0.1) \times 10^{-10}$ \\
\hline \multirow[t]{5}{*}{ NтнСомр } & $\begin{array}{l}\Gamma \\
k T_{\mathrm{e}}(\mathrm{keV}) \\
k T_{\mathrm{bb}}(\mathrm{keV})\end{array}$ & $\begin{array}{c}2.6_{-0.5}^{+0.3} \\
>7 \\
1.3_{-0.1}^{+0.2}\end{array}$ & $\begin{array}{c}1.5_{-0.3}^{+0.5} \\
5_{-1}^{+3} \\
1.10_{-0.3}^{+0.09}\end{array}$ & $\begin{array}{c}2.2_{-0.3}^{+0.7} \\
\geq 1.2 \\
1.9_{-0.4}^{+0.2}\end{array}$ & $\begin{array}{c}1.5_{-0.3}^{+0.5} \\
5_{-1}^{+2} \\
1.11_{-0.2}^{+0.03}\end{array}$ \\
\hline & inp_type $(0 / 1)$ & 0 & $0^{-0.3}$ & $0^{-0.4}$ & $0^{-0.2}$ \\
\hline & Redshift & 0 & 0 & 0 & 0 \\
\hline & Norm. $\left(10^{-3}\right)$ & $5_{-3}^{+1}$ & $2_{-1}^{+5}$ & $<3.4$ & $<11.8$ \\
\hline & NthComp Unabs. Flux $\left(\mathrm{erg} \mathrm{cm}^{2} \mathrm{~s}^{-1}\right)$ & $(3.8 \pm 0.4) \times 10^{-10}$ & $(2.9 \pm 0.3) \times 10^{-10}$ & $<3 \times 10^{-10}$ & $<4 \times 10^{-10}$ \\
\hline \multirow[t]{7}{*}{ NтнСомР } & $\Gamma$ & - & - & $2.2_{-0.3}^{+0.7}$ & $1.5_{-0.3}^{+1}$ \\
\hline & $k T_{\mathrm{e}}(\mathrm{keV})$ & - & - & $>1$ & $5_{-1}^{+-2.3}$ \\
\hline & $k T_{\mathrm{bb}}(\mathrm{keV})$ & - & - & $0.60_{-0.1}^{+0.07}$ & $0.55_{-0.04}^{+0.05}$ \\
\hline & inp_type $(0 / 1)$ & - & - & $1^{-0.1}$ & $1^{-0.04}$ \\
\hline & Redshift & - & - & 0 & 0 \\
\hline & Norm. $\left(10^{-3}\right)$ & - & - & $41_{-21}^{+22}$ & $<31$ \\
\hline & NthComp Unabs. FluX $\left(\mathrm{erg} \mathrm{cm}^{2} \mathrm{~s}^{-1}\right)$ & - & - & $(3.4 \pm 0.3) \times 10^{-10}$ & $<4 \times 10^{-10}$ \\
\hline \multirow{5}{*}{$\begin{array}{l}\text { Gaussian } \\
(\mathrm{Fe} \operatorname{xxv} \mathrm{K} \alpha)\end{array}$} & LineE $(\mathrm{keV})$ & $6.65 \pm 0.03$ & $6.69_{-0.02}^{+0.03}$ & $6.65 \pm 0.03$ & $6.69 \pm 0.02$ \\
\hline & Sigma (keV) & 0.02 (frozen) & 0.02 (frozen) & 0.02 (frozen) & 0.02 (frozen) \\
\hline & Norm $\left(10^{-5}\right)$ & $-3 \pm 1$ & $-3.9_{-07}^{+0.6}$ & $-3 \pm 1$ & $-3.9 \pm 0.7$ \\
\hline & DETECTION SIGNIFICANCE $(\sigma)$ & 4 & 9 & 4 & 10 \\
\hline & EQ. WIDTH $(\mathrm{keV})$ & $-0.011 \pm 0.003$ & $-0.005 \pm 0.002$ & $-0.014 \pm 0.003$ & $-0.013 \pm 0.002$ \\
\hline \multirow{5}{*}{$\begin{array}{l}\text { GaUSSIAN } \\
(\mathrm{Fe} \text { xxvI K } \alpha \text { ) }\end{array}$} & LineE $(\mathrm{keV})$ & $6.943_{-0.013}^{+0.02}$ & $6.972_{-0.010}^{+0.014}$ & $6.943_{-0.013}^{+0.02}$ & $6.974_{-0.011}^{+0.012}$ \\
\hline & Sigma (keV) & 0.02 (frozen) & 0.02 (frozen) & 0.02 (frozen) & 0.02 (frozen) \\
\hline & Norm $\left(10^{-5}\right)$ & $-8 \pm 1$ & $-6.8 \pm 0.7$ & $-8 \pm 1$ & $-6.8_{-0.6}^{+0.7}$ \\
\hline & DETECTION SIGNIFICANCE $(\sigma)$ & 11 & 17 & 10 & 17 \\
\hline & EQ. WIDTH $(\mathrm{keV})$ & $-0.029 \pm 0.003$ & $-0.017 \pm 0.002$ & $-0.033 \pm 0.003$ & $-0.026 \pm 0.002$ \\
\hline \multirow[t]{4}{*}{$\begin{array}{l}\text { Gaussian } \\
(\mathrm{Fe} x \mathrm{xx} \mathrm{K} \beta)\end{array}$} & $\begin{array}{l}\text { LineE }(k e V) \\
\text { Sigma }(k e V)\end{array}$ & $\underset{0.02 \text { (frozen) }}{-}$ & $\begin{array}{c}7.79 \pm 0.08 \\
0.02 \text { (frozen) }\end{array}$ & $\underset{0.02 \text { (frozen) }}{-}$ & $\begin{array}{c}7.79_{-0.04}^{+0.08} \\
0.02 \text { (frozen) }\end{array}$ \\
\hline & Norm $\left(10^{-5}\right)$ & - & $-1.3 \pm 0.7$ & - & $-1.3_{-04}^{+0.6}$ \\
\hline & DETECTION SIGNIFICANCE $(\sigma)$ & - & 3 & - & $4^{-0.4}$ \\
\hline & EQ. WIDTH $(\mathrm{keV})$ & - & $-0.005 \pm 0.003$ & - & $-0.006 \pm 0.003$ \\
\hline & LineE $(\mathrm{keV})$ & - & $8.19 \pm 0.07$ & - & $8.19 \pm 0.07$ \\
\hline \multirow[t]{6}{*}{$(\mathrm{Fe}$ xxvI K $\beta)$} & Sigma (keV) & 0.02 (frozen) & 0.02 (frozen) & 0.02 (frozen) & 0.02 (frozen) \\
\hline & $\operatorname{Norm}\left(10^{-5}\right)$ & $\begin{array}{c}-02(102011) \\
-\end{array}$ & $-1.7 \pm 0.7$ & 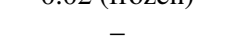 & $-1.7 \pm 0.7$ \\
\hline & DETECTION SIGNIFICANCE $(\sigma)$ & - & 4 & - & 4 \\
\hline & EQ. WIDTH $(\mathrm{keV})$ & - & $-0.005 \pm 0.003$ & - & $-0.010 \pm 0.003$ \\
\hline & Total Unabs. Flux $\left(\times 10^{-10} \mathrm{erg} \mathrm{cm}^{2} \mathrm{~s}^{-1}\right)$ & $5.8 \pm 0.6$ & $5.3 \pm 0.5$ & $5.8 \pm 0.6$ & $5.4 \pm 0.5$ \\
\hline & $\chi^{2} /$ d.o.f. & $702.5 / 617$ & $693.6 / 600$ & $698.0 / 616$ & $693.5 / 599$ \\
\hline
\end{tabular}

Notes. The errors for each spectral parameter are quoted at the $90 \%$ of statistical confidence.

we combined the XIS0 and XIS3 spectra (hereafter XIS03 spectrum) and each HXD/PIN spectrum of the two observations to further increase the statistic of the observations. We obtained a broadband spectrum with a considerably high statistics: the resulting exposure times for the XIS03 and PIN total combined spectra are of $400 \mathrm{ks}$ and $9.3 \mathrm{ks}$, respectively. 
Table 2. Results of the fit on Spectrum 1 and Spectrum 2, adopting the 1-seed and 2-seed models together with a component that takes into account the contribution of a local warm absorber, respectively.

\begin{tabular}{|c|c|c|c|c|c|}
\hline \multirow[b]{2}{*}{ Component } & \multirow[b]{2}{*}{ Parameter } & \multicolumn{2}{|c|}{ Model: warm_1seed } & \multicolumn{2}{|c|}{ Model: warm_2seed } \\
\hline & & Spectrum 1 & Spectrum 2 & Spectrum 1 & Spectrum 2 \\
\hline PHABS & $N_{\mathrm{H}}\left(10^{22}\right)$ & $0.432_{-0.009}^{+0.007}$ & $0.47 \pm 0.02$ & $0.46 \pm 0.02$ & $0.47 \pm 0.02$ \\
\hline \multirow[t]{4}{*}{ ZXIPCF } & $N_{\mathrm{H}}\left(10^{22}\right)$ & $160 \pm 120$ & $13_{-3}^{+8}$ & $140_{-110}^{+130}$ & $13_{-3}^{+8}$ \\
\hline & $\log (\xi)$ & $5.2_{-0.5}^{+0.4}$ & $4.35_{-0.04}^{+0.20}$ & $5.1 \pm 0.5$ & $4.35_{-0.04}^{+0.2}$ \\
\hline & CvrFract & $>0.87$ & 1 & $>0.87$ & $>0.97$ \\
\hline & Redshift & 0 & 0 & 0 & 0 \\
\hline \multirow[t]{3}{*}{ GABS } & LineE $(\mathrm{keV})$ & - & $4.38_{-0.11}^{+0.12}$ & - & $4.38_{-0.10}^{+0.11}$ \\
\hline & Sigma(keV) & - & $0.72_{-0.14}^{+0.2}$ & - & $0.72 \pm 0.2$ \\
\hline & Strength & - & $0.07_{-0.03}^{+0.05}$ & - & $0.07_{-0.02}^{+0.04}$ \\
\hline \multirow[t]{2}{*}{ EDGE } & edgeE (keV) & - & $0.870_{-0.012}^{+0.014}$ & - & $0.869_{-0.012}^{+0.014}$ \\
\hline & MaxTau & - & $0.11_{-0.02}^{+0.03}$ & - & $0.113_{-0.02}^{+0.03}$ \\
\hline \multirow[t]{3}{*}{ BBODY } & $k T(\mathrm{keV})$ & $1.12_{-0.11}^{+0.05}$ & $0.750_{-0.010}^{+0.20}$ & $2.0 \pm 0.3$ & $0.72_{-0.06}^{+0.7}$ \\
\hline & Norm. $\left(10^{-5}\right)$ & $<45$ & $\leq 50$ & $\leq 128$ & $\leq 20$ \\
\hline & BBody UnABs. FluX $\left(\mathrm{erg} \mathrm{cm}^{2} \mathrm{~s}^{-1}\right)$ & $<4 \times 10^{-11}$ & $<4 \times 10^{-11}$ & $<1 \times 10^{-10}$ & $<2 \times 10^{-11}$ \\
\hline \multirow[t]{3}{*}{ DISKBB } & $T_{\text {in }}(\mathrm{keV})$ & $0.69_{-0.05}^{+0.04}$ & $0.51_{-0.06}^{+0.04}$ & $0.52_{-0.6}^{+0.11}$ & $0.50 \pm 0.05$ \\
\hline & $\left(R_{\mathrm{in}} / D_{10}\right)^{2} \cos (\theta)\left(\mathrm{km}^{2} \mathrm{kpc}^{-2}\right)$ & $47_{-7}^{+13}$ & $78_{-13}^{+34}$ & $<28$ & $82_{-9}^{+270}$ \\
\hline & DISKBb UnABs. FluX $\left(\mathrm{erg} \mathrm{cm}^{2} \mathrm{~s}^{-1}\right)$ & $(2.0 \pm 0.2) \times 10^{-10}$ & $(1.1 \pm 0.1) \times 10^{-10}$ & $<6 \times 10^{-11}$ & $(1.1 \pm 0.1) \times 10^{-10}$ \\
\hline \multirow[t]{7}{*}{ NтнСомР } & $\Gamma$ & $2.1_{-0.2}^{+0.3}$ & $1.84_{-0.05}^{+0.04}$ & $2.4 \pm 0.5$ & $1.8 \pm 0.4$ \\
\hline & $k T_{\mathrm{e}}(\mathrm{keV})$ & $7 \pm 1$ & $6 \pm 1$ & $12_{-9}^{+27}$ & $6 \pm 1$ \\
\hline & $k T_{\mathrm{bb}}(\mathrm{keV})$ & $1.12_{-0.11}^{+0.05}$ & $0.750_{-0.010}^{+0.2}$ & $2.0 \pm 0.3$ & $0.72_{-0.06}^{+0.7}$ \\
\hline & inp_type $(0 / 1)$ & $0^{-0.11}$ & 0 & 0 & $0^{-0.00}$ \\
\hline & Redshift & 0 & 0 & 0 & 0 \\
\hline & Norm. $\left(10^{-3}\right)$ & $6.0_{-0.5}^{+2}$ & $10_{-3}^{+12}$ & $<2.1$ & $11.2_{-7}^{+3}$ \\
\hline & NтнCомP Unabs. FluX $\left(\mathrm{erg} \mathrm{cm}^{2} \mathrm{~s}^{-1}\right)$ & $(4.0 \pm 0.4) \times 10^{-10}$ & $(4.0 \pm 0.4) \times 10^{-10}$ & $<3 \times 10^{-10}$ & $(4.0 \pm 0.4) \times 10^{-10}$ \\
\hline \multirow[t]{9}{*}{ NтнСомР } & $\Gamma$ & - & - & $2.4 \pm 0.5$ & $1.8 \pm 0.4$ \\
\hline & $k T_{\mathrm{e}}(\mathrm{keV})$ & - & - & $12_{-9}^{+27}$ & $6 \pm 1$ \\
\hline & $k T_{\mathrm{bb}}(\mathrm{keV})$ & - & - & $0.52_{-0.6}^{+0.11}$ & $0.50 \pm 0.05$ \\
\hline & inp_type $(0 / 1)$ & - & - & $1^{-0.0}$ & 1 \\
\hline & Redshift & - & - & 0 & 0 \\
\hline & Norm. $\left(10^{-3}\right)$ & - & - & $61_{-21}^{+6}$ & $<8$ \\
\hline & NThComp Unabs. Flux $\left(\mathrm{erg} \mathrm{cm}^{2} \mathrm{~s}^{-1}\right)$ & - & - & $(4.0 \pm 0.4) \times 10^{-10}$ & $<9 \times 10^{-11}$ \\
\hline & TOtal Unabs. FluX $\left(\times 10^{-10} \mathrm{erg} \mathrm{cm}^{2} \mathrm{~s}^{-1}\right)$ & $6.0 \pm 0.6$ & $5.1 \pm 0.5$ & $6.0 \pm 0.6$ & $5.1 \pm 0.5$ \\
\hline & $\chi^{2} /$ d.o.f. & $698.30 / 618$ & $707.51 / 605$ & $691.30 / 617$ & $707.48 / 604$ \\
\hline
\end{tabular}

Notes. The errors for each spectral parameter are quoted at $90 \%$ of statistical confidence.

\section{Data analysis}

\subsection{Persistent spectrum}

The broadband spectrum obtained from Obs 1, hereafter named Spectrum 1, covers the energy range between $0.8 \mathrm{keV}$ and $40 \mathrm{keV}$. Similarly, the spectrum obtained from the combination of data of Obs 2 and Obs 3 is named Spectrum 2 and covers the energy range between $0.8 \mathrm{keV}$ and $30 \mathrm{keV}$. In these two spectra, however, the XIS data cover the energy range between $0.8 \mathrm{keV}$ and $10 \mathrm{keV}$, whereas the data of the HXD/PIN cover the energy range between $15 \mathrm{keV}$ and $40 \mathrm{keV}$ in Spectrum 1 and between $15 \mathrm{keV}$ and $30 \mathrm{keV}$ in Spectrum 2, respectively. We ignored the energy ranges $1.7-1.9 \mathrm{keV}$ and $2.2-2.4 \mathrm{keV}$ in all the XIS spectra to exclude calibration uncertainties due to the presence of the instrumental K-edge of silicon and M-edge of gold, respectively.
In order to avoid an oversampling of the energy resolution of the XIS, we applied a grouping of a factor 4 to the data. Furthermore, we grouped all the spectra to have at least 25 photons per energy channel and to use the $\chi^{2}$ as an estimator of the goodness of the fit. The only exception to this is for the HXD/PIN data of Spectrum 1, where because of the lower statistics we grouped the spectrum to have at least 100 photons per energy channel.

In the subsequent part of this work we want to use the model adopted by Zhang et al. (2014) to describe the persistent spectrum of XB 1916-053, taking the advantage of the whole available Suzaku dataset. We seek to study the relative contribution of each spectral component with respect to the total observed flux, and we also compare this model with a simpler model that takes into account a single source of photons for the Comptonization that is represented by photons emitted by the NS surface. 
Moreover, we aim to study the emission features that are visible in the available spectra, trying to characterize the chemical abundances of the species from which they are produced. To complete this task, as a first step we decided to adopt the simpler model to describe the continuum emission of the source.

For the spectral analysis we used Xspec v. 12.9.1 and the cross-section table of Verner et al. (1996) and the chemical abundances reported by Wilms et al. (2000).

We first fitted Spectrum 1 with a spectral model that is usually suitable for atoll class LMXB systems (see, e.g., di Salvo et al. 2009; Piraino et al. 2007). This model consists of a soft blackbody component plus a multicolor-disk blackbody and a hard component usually modeled with a Comptonization spectrum.

More precisely, to take into account the contribution of emission by the NS surface we used the bbody model that is defined by a temperature $k T$ and a normalization. The disk contribution was modeled using the multicolor-disk blackbody component (diskbb, see Mitsuda et al. 1984) that consists of two parameters: the temperature at the inner disk radius and a normalization parameter $N$ linked to the value of the inner radius through the relation $N=\left(R_{\text {in }} / D_{10}\right)^{2} \cos (\theta)$, where $R_{\text {in }}$ is an apparent inner disk radius in $\mathrm{km}, D_{10}$ is the distance to the source in units of $10 \mathrm{kpc}$, and $\theta$ is the inclination angle of the disk with respect to the line of sight $(\theta=0$ is the face-on case). The contribution of a thermally Comptonized continuum was included by adopting the model nthComp (Życki et al. 1999). This is defined by an asymptotic power-law photon index $\Gamma$ and by the temperatures $k T_{\mathrm{e}}$ and $k T_{\mathrm{bb}}$ of the electron cloud and seed photons, respectively. This model also takes into account a redshift parameter and the parameter inp_type that can be set to 0 or 1 , depending on the seed photons distribution as a blackbody or disk blackbody, respectively. As a first step, we assume that the photons that are subsequently Comptonized are those emitted by the NS surface, imposing inp_type $=0$. On the other hand, to take into account the photoelectric absorption by the neutral matter in the interstellar medium (ISM), we used the multiplicative spectral component phabs, defined through the parameter $N_{\mathrm{H}}$ that is the equivalent hydrogen column density (in units of $10^{22}$ atoms $\mathrm{cm}^{-2}$ ). To take into account the different instrumental responses of the XIS and HXD/PIN detectors we also introduced a multiplicative intercalibration constant into the model.

The application of this model (hereafter 1-seed model) to Spectrum 1 returns a $\chi^{2}$ (d.o.f.) of 818.11(621) and evident residuals in absorption at about $7 \mathrm{keV}$, as observed by Zhang et al. (2014). We modeled these residuals with the two absorption lines already found by Boirin et al. (2004) using the additive spectral component gauss in Xspec. We fixed the value of the line widths to $\sigma=20 \mathrm{eV}$ due to the moderate energy resolution of the Suzaku/XIS instrument. This value is compatible with the line width of the Fe xxvI K $\alpha$ absorption line evaluated by Iaria et al. (2006) using data of the Chandra observatory, and this value has been used for all the subsequent analysis.

The fit returned a value of $\chi^{2}$ (d.o.f.) of 702.5(617) and an $F$-test probability of chance improvement of $1.7 \times 10^{-19}$ with respect to the previous model. We obtained two lines centered at 6.65 and $6.943 \mathrm{keV}$, actually compatible with the $\mathrm{K} \alpha$ absorption lines of Fe Xxv and Fe xxvI, respectively. The detection of these absorption lines was obtained with a confidence level of $4 \sigma$ and $11 \sigma$, respectively, and the obtained fit parameters are reported in Table 1.

Finally, from this best-fit model we extrapolated an unabsorbed flux of $(5.8 \pm 0.6) \times 10^{-10} \mathrm{erg} \mathrm{cm}^{-2} \mathrm{~s}^{-1}$ in the $0.1-100 \mathrm{keV}$ energy band. The bbody component provides a marginal contribution to the total flux since it contributes with less than the $14 \%$ with respect to the total. On the other hand, the spectral components diskbb and nthComp contribute to the $35 \%$ and $65 \%$, respectively.

In order to understand if substantial changes in the spectral shape of the source occurred between the observations of 2006 and 2014, we fitted Spectrum 2 with the same model. The fit returned a value of $\chi^{2}$ (d.o.f.) of 693(600) and evident residuals in absorption at about $8 \mathrm{keV}$, probably owing to the combination of more than one absorption line. The residuals also show a bump at about $4.5 \mathrm{keV}$ and the clear presence of an edge at about $0.8 \mathrm{keV}$, that is the energy at which the $\mathrm{K}$ absorption edge of $\mathrm{O}$ VIII $(E \sim 0.871 \mathrm{keV})$ is expected. Then, we modeled this last feature with an absorption edge at $0.871 \mathrm{keV}$, while the absorption feature at about $8 \mathrm{keV}$ was modeled with two Gaussian absorption lines with a fixed width of $\sigma=20 \mathrm{eV}$. The bump in the residuals at $4.5 \mathrm{keV}$ is often observed in high statistics Suzaku/XIS spectra and it is probably of systematic and nonphysical nature. To get rid of this feature, we modeled it with the spectral component gabs.

The detection of the two $\mathrm{K} \alpha$ lines remains confirmed as in Spectrum 1, but we find that they are stronger in Spectrum 2 because of its higher statistics. Indeed, the spectral model returned a detection of the Fexxv and Fexxvi $\mathrm{K} \alpha$ lines with a level of confidence of about $9 \sigma$ and $17 \sigma$, respectively. In addition, the two supplementary absorption lines added returned energies that are consistent with those we expect for the $\mathrm{K} \beta$ absorption lines of Fexxv and Fexxvi. Our fit allowed us to detect these lines with a level of confidence of about $3 \sigma$ and of $4 \sigma$, respectively.

At last, the model returned a value of unabsorbed flux of $(5.3 \pm 0.5) \times 10^{-10} \mathrm{erg} \mathrm{cm}^{-2} \mathrm{~s}^{-1}$ between 0.1 and $100 \mathrm{keV}$, to which contribute the bbody component for about the $22 \%$, the diskbb component for the $24 \%$, and the nthcomp component for about the $55 \%$ of the total flux.

The results obtained from the application of this simple model both to Spectrum 1 and Spectrum 2 are typical of sources in a soft spectral state. Adopting a distance to the source of $9.3 \mathrm{kpc}$ (Yoshida 1993), we obtained that the luminosity of XB 1916-053 is about the $2 \%$ of the Eddington luminosity. According to this, taking into account the color correction factor $\psi=1.7$ of Shimura \& Takahara (1995) we obtained the inner radius of the accretion disk as $R_{\text {disk }}=\sqrt{\text { norm } / \cos (\theta)} \psi^{2} D_{10}$, where norm is the normalization parameter of the diskbb component, $\theta$ is the inclination angle of the system, and $D_{10}$ is the distance to the source in units of $10 \mathrm{kpc}$. The resulting inner radius of the accretion disk seems to be considerably close to the compact object in both the analyzed spectra, i.e., on the order of $30 \pm 10 \mathrm{~km}$ and $38_{-13}^{+14} \mathrm{~km}$ for Spectrum 1 and 2, respectively.

These values of the inner radius of the accretion disk are in agreement with those estimated by Zhang et al. (2014). As suggested by these authors, since the disk is so close to the NS surface it is possible that the electron corona Compton-scatters a fraction of the photons emitted by the innermost part of the accretion disk in addition to the photons emitted by the NS surface. Zhang et al. (2014) tested this hypothesis by adopting a double Comptonization model to fit the persistent spectrum of XB 1916-053. Considering the possibility of this phenomenology, as a further step we reapplied this model to the spectrum already studied by Zhang et al. (2014) (i.e., Spectrum 1), and for the first time to the composite high statistic Spectrum 2.

The model consists of a further thermal Comptonization component with respect to the case of the 1-seed model. While 
the first component (nthComp[inp_type=0]) scatters photons distributed following a blackbody law, this second component (nthComp[inp_type=1]) scatters photons that are emitted by the accretion disk and are distributed following a multicolordisk blackbody law. Assuming that all these photons are scattered by the same electron cloud, we kept tied the values of the photon index $\Gamma$ and electron temperature $k T_{\mathrm{e}}$ for these two Comptonization components. On the other hand, we imposed that the temperature $k T_{\mathrm{bb}}$ for (nthComp[inp_type=0] and (nthComp[inp_type $=1]$ was the same of the bbody and diskbb components, respectively. We also included all the absorption lines we detected using the 1-seed model, leaving the associated energies free to vary and keeping fixed their width to the same value adopted in the first part of the data analysis, that is $\sigma=20 \mathrm{eV}$.

Fitting this model (hereafter 2-seed model) on Spectrum 1 we obtained a good accordance with the results provided by using the 1-seed model. We extrapolated a total unabsorbed flux of $(5.8 \pm 0.6) \times 10^{-10} \mathrm{erg} \mathrm{cm}^{-2} \mathrm{~s}^{-1}$ in the energy band $0.1-100 \mathrm{keV}$. The bbody component contributes less than $17 \%$ to the total flux, while the diskbb, nthComp [inp_type=0] and nthComp[inp_type=1] components contribute less than $16 \%, 52 \%$, and $59 \%$, respectively (see the best-fit parameters in Table 1).

We confirm the detection of the $\mathrm{K} \alpha$ absorption lines of Fe xxv and Fe xxvi with a level of statistical confidence of $4 \sigma$ and $10 \sigma$, respectively.

The temperature of the seed photons emitted from the NS surface is of about $k T \sim 2 \mathrm{keV}$, while that related to the accretion disk is of about $k T_{\mathrm{bb}}=0.6 \mathrm{keV}$. In addition, the fit returns a photon index for the corona that is equal to $\Gamma=2.21$ and a lower limit on the electron temperature of $k T_{\mathrm{e}}>1 \mathrm{keV}$. These parameters are all in agreement with those found using the 1-seed model to fit the spectrum and support the hypothesis that the source could probably be in a soft spectral state, as already suggested by Zhang et al. (2014). The fit, however, returned a value of the $\chi^{2}$ (d.o.f.) of 698(616), which does not represent a statistically significant improvement with respect to the 1-seed model because of the $F$-test probability that the improvement of the fit occurs by chance on the order of the $5 \%$.

We also considered the case in which the corona that envelopes the NS has a different optical depth (and then a different $\Gamma$ index) with respect to the corona that covers the inner regions of the accretion disk. However, we imposed that these coronae have the same electron temperature $\left(k T_{\mathrm{e}}\right)$. The bestfit parameters are fully in agreement with those obtained with the previous model and we obtained a $\chi^{2}$ (d.o.f.) of 696.5(615) with an $F$-test probability of chance improvement of about the $3 \%$. With a such a poor outcome for the $F$-test, we did not consider the improvement to the fit statistically significant and we therefore decided not to take a double- $\Gamma$ index corona into account.

Applying the 2-seed model to the higher statistics Spectrum 2 as well, we obtained results that are consistent with those obtained fitting this model onto the Spectrum 1, suggesting that the continuum emission of XB 1916-053 is compatible in the two observations that are separated by a time interval of eight years.

We extrapolated a total unabsorbed flux of $(5.4 \pm 0.5) \times 10^{-10}$

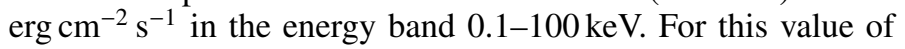
flux the bbody component contributes for less than the $56 \%$, while the diskbb component contributes about $24 \%$ of the flux and the nthComp[inp_stype $=0]$ and nthComp[inp_type $=1]$ components less than $74 \%$ each, respectively. We also detected the
$\mathrm{K} \alpha$ absorption lines of Fe Xxv and Fe XXvI in this case with a level of statistical confidence of 10 and $17 \sigma$, respectively. The $\mathrm{K} \beta$ absorption lines of Fexxv and Fe xxvI, on the other hand, were detected with a level of confidence of about $4 \sigma$.

Similar to the case in which we fitted the 1-seed model to Spectrum 2, in this case we detected an edge at $0.877 \mathrm{keV}$ that is compatible with a $\mathrm{O}$ vIII $\mathrm{K}$ absorption edge. The fit, however, returned a $\chi^{2}$ (d.o.f.) of 693.5(599) that actually does not represent a significant improvement of the fit performed on the Spectrum 1 with the same model, since the $F$-test probability of chance improvement is of about $99 \%$. Moreover, this fit does not represent an improvement with respect to the 1-seed model applied to the same spectrum, since the $F$-test probability of chance improvement is about $77 \%$.

As already considered for the Spectrum 1, we tried to take into account different values of the index $\Gamma$ for the two Comptonization components, assuming the same electron temperature. Also in this case, the fit was not sensitive to the change into the spectral model and returned a $\chi^{2}$ (d.o.f.) of 693.5(598) with an $F$-test probability that the improvement occurs by chance of $100 \%$ with respect to the previous fit.

From a comparison between the two adopted models, it is possible to notice a certain indetermination in the evaluation of the goodness of the 2-seed model with respect to the single seed model. The $F$-test probability that the improvement introduced by the adoption of the 2-seed (with respect to the 1-seed model) occurs by chance is equal to $1 \%$ in the case of the lower statistic Spectrum 1 and to $77 \%$ for Spectrum 2.

The presence of several absorption lines due to the presence of different iron ions, however, suggests the possibility that a local partially ionized absorber is located between the observer and the system, as already suggested by Boirin et al. (2005). To verify this scenario, we modified the 1 -seed and 2 -seed models adding the multiplicative spectral component zxipcf and getting rid of the Gaussian components used to model the absorption lines and the edge. Hereafter, we call warm_1seed model the 1-seed model to which we applied the zxipcf component, while the application of the same component to the 2-seed model results in a model that is named warm_2seed model.

The zxipcf component uses a grid of XSTAR photoionized absorption models to describe the absorption of the incoming radiation by the plasma, taking into account a micro-turbulent velocity of the plasma of $200 \mathrm{~km} / \mathrm{s}$. The model assumes that the absorbing plasma covers only a fraction of the source, whilst the remaining part of the spectrum is seen directly (see Reeves et al. 2008; Miller et al. 2007). Moreover, this model takes into account the column density of absorbing material $N_{\mathrm{H}}$, the ionization state of the plasma $\log (\xi)$, and the covering fraction and redshift. The results of the fits reported in Table 2, show similar and compatible values with respect to those obtained with the 1-seed and 2-seed models.

The unabsorbed flux extrapolated in the band $0.1-100 \mathrm{keV}$ by fitting Spectrum 1 and Spectrum 2 with the model warm_1seed is equal to $(6.0 \pm 0.6) \times 10^{-10} \mathrm{erg} \mathrm{cm}^{-2} \mathrm{~s}^{-1}$ and $(5.1 \pm 0.5) \times 10^{-10} \mathrm{erg} \mathrm{cm}^{-2} \mathrm{~s}^{-1}$, respectively. We extrapolated the same fluxes adopting the warm_2seed model to the same observations (see Table 2).

Adopting the warm_1seed model, the contribution of the blackbody component is quite small, since this component contributes with less than $7 \%$ and $8 \%$ to the total flux in Spectrum 1 and Spectrum 2, respectively. In addition, the diskbb component contributes $33 \%$ and $22 \%$ of the flux, while the nthComp[inp_type=0] contributes $67 \%$ and $77 \%$ for Spectrum 1 and Spectrum 2, respectively. On the other hand, 

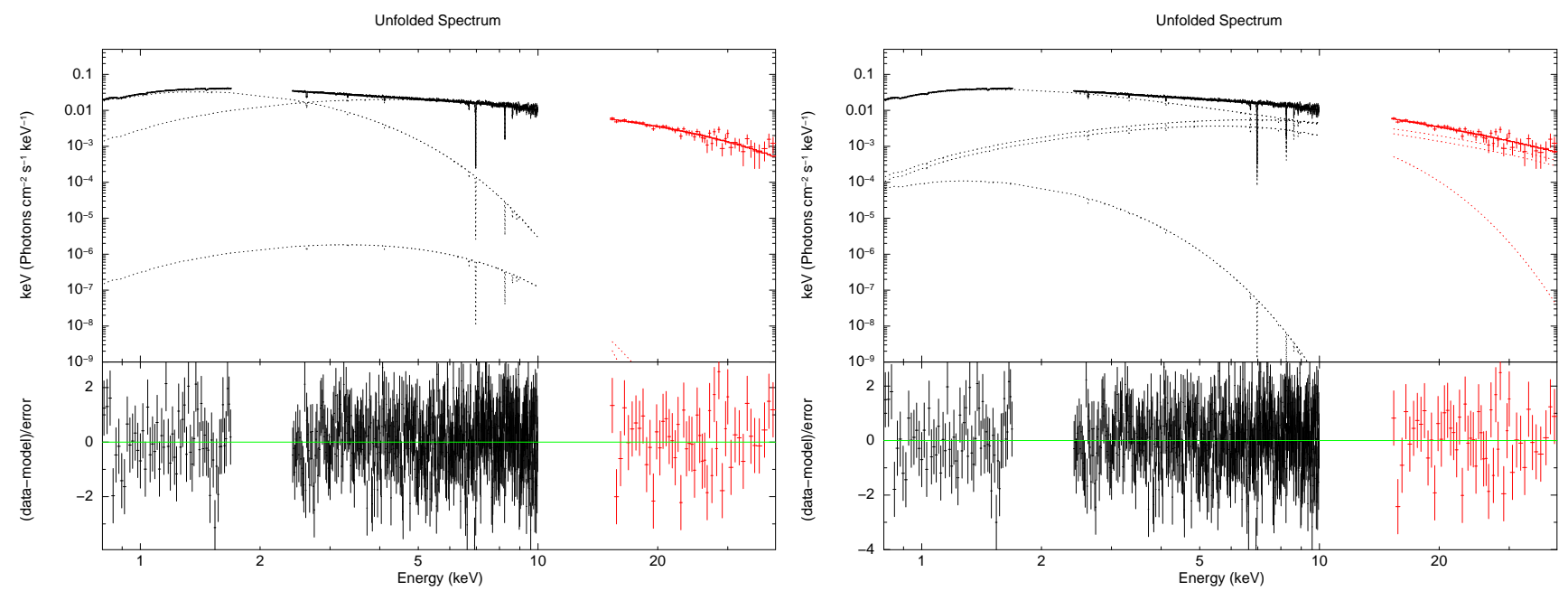

Fig. 2. Best-fit models performed on data of ObsID 401095010 (Spectrum 1) taking into account the contribution of a local absorber. Left: warm_1seed model. Right: warm_2seed model. In each plot the black data represent the XIS03 spectrum, whilst the red data represent the PIN spectrum. The bottom panel of each plot represents the residuals in units of sigma with respect to the adopted model.
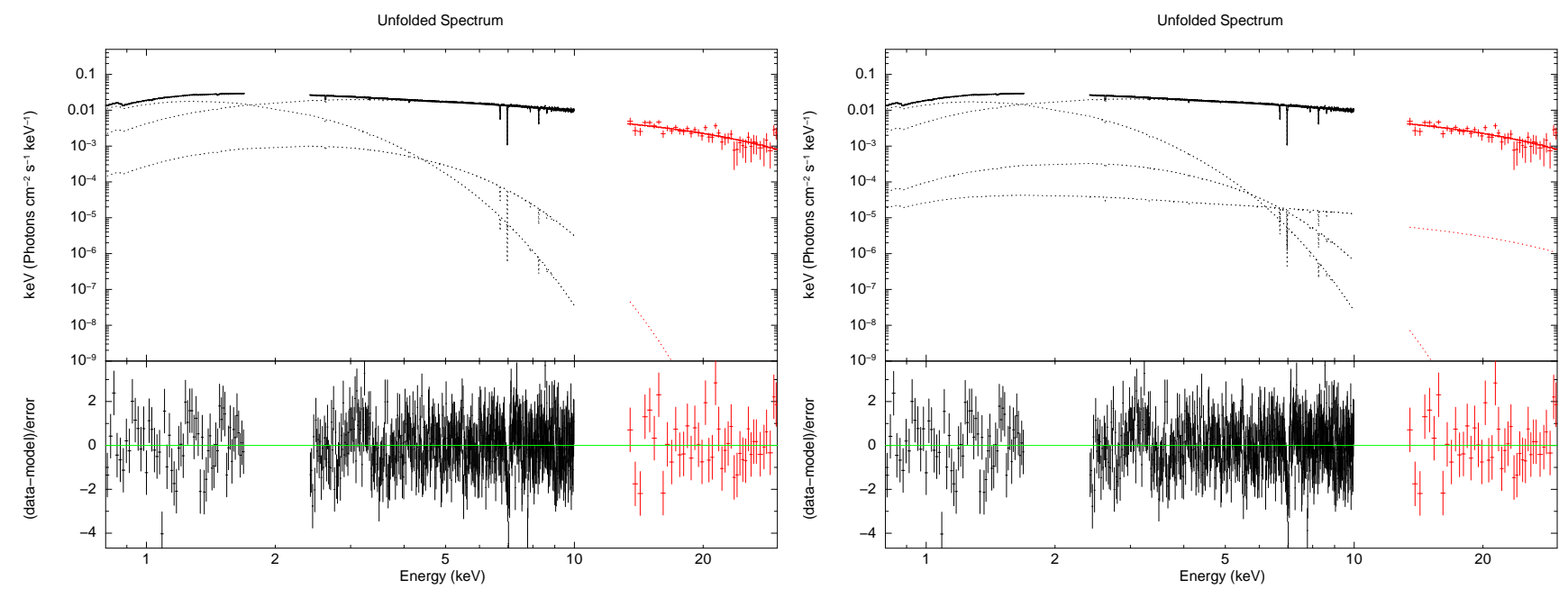

Fig. 3. Best-fit models performed on data of ObsID $409032010+409032020$ (Spectrum 2) taking into account the contribution of a local absorber. Left: warm_1seed model. Right: warm_2seed model. In each plot the black data represent the combined XIS03 spectra of the two observations, whilst the red data represent the combined PIN spectra of the two observations. The bottom panel of each plot represents the residuals in units of sigma with respect to the adopted model.

adopting the warm_2seed model we notice that the blackbody component contributes with less than $17 \%$ and $4 \%$ for Spectrum 1 and Spectrum 2, while the diskbb component contributes with less than $10 \%$ for Spectrum 1 and 22\% Spectrum 2. Moreover, the nthComp [inp_type $=0$ ] contributes with less than $50 \%$ for Spectrum 1 and $79 \%$ for Spectrum 2, while the nthComp [inp_type $=1$ ] component contributes $67 \%$ of the flux for Spectrum 1 and less than $18 \%$ for Spectrum 2.

The addition of the absorption component due to a warm partially ionized absorber well fits the absorption line shapes and returns values of the $\chi^{2}$ (d.o.f.) of 698.3(618) and 707.51(605) for the warm_1seed model applied to Spectrum 1 and Spectrum 2, respectively, and of 691.3(617) and 707.48(604) for the warm_2seed model applied to Spectrum 1 and Spectrum 2 , respectively. Also in this case, the improvement introduced by the adoption of the warm_2seed model with respect to the warm_1seed is not statistically significant, owing to the $F$-test probability of chance improvement that is equal to $1 \%$ and $87 \%$ for Spectrum 1 and Spectrum 2, respectively; this is exactly what occurred in the case of the 1-seed and 2-seed models. According to the case of the Spectrum 2, this result could actually reflect the poor contribution of the nthComp[inp_type $=1$ ] component in terms of flux (the $<18 \%$ of the total flux) with respect to the nthComp[inp_type $=0$ ] component, which provides a higher contribution of flux ( $79 \%$ of the total flux). In Fig. 2 and 3 we show the best-fit models warm_1seed and warm_2seed applied to Spectrum 1 and Spectrum 2 , respectively, with the relative residuals.

Even though it is not possible to break the indetermination of the adopted models, in the following we discuss the parameters obtained by fitting the warm_1seed model on Spectrum 2, that is the spectrum with higher statistics, preferring a less parameterized model.

\subsection{Dip spectrum}

While the persistent emission spectrum of XB 1916-053 does not show spectral changes between the Obs 2 and Obs 3, on the other hand, the dip spectrum appears to be inconsistent between these two observations, in particular at the low energies. As a 
Table 3. Results of the fit on the dip spectra of Obs 2 and Obs 3, adopting the 1-seed model together with the contribution of two local warm absorbers, respectively.

\begin{tabular}{llcc}
\hline \hline Model & Component & Obs 2 & Obs 3 \\
\hline PHABS & $N_{\mathrm{H}}\left(10^{22}\right)$ & 0.479 (frozen) & 0.479 (frozen) \\
CABS & $N_{\mathrm{H}}\left(10^{22}\right)$ & $15_{-3}^{+2}$ & $16 \pm 1$ \\
CABS & $N_{\mathrm{H}}\left(10^{22}\right)$ & $67 \pm 4$ & $91_{-4}^{+6}$ \\
zXIPCF & $N_{\mathrm{H}}\left(10^{22}\right)$ & $15_{-3}^{+2}$ & $17 \pm 1$ \\
& $\log (\xi)$ & $0.7_{-0.6}^{+0.5}$ & $1.14_{-0.3}^{+0.14}$ \\
& CvrFract & $0.80 \pm 0.03$ & $0.891_{-0.012}^{+0.010}$ \\
& Redshift & 0 (frozen) & 0 (frozen) \\
zXIPCF & $N_{\mathrm{H}}\left(10^{22}\right)$ & $67 \pm 4$ & $91_{-4}^{+6}$ \\
& $\log (\xi)$ & $3.24_{-0.13}^{+0.2}$ & $3.05 \pm 0.12$ \\
& CvrFract & $0.64_{-0.13}^{+0.12}$ & $0.57_{-0.10}^{+0.09}$ \\
& Redshift & 0 (frozen) & 0 (frozen) \\
BBODY & $k T$ (keV) & 0.75 (frozen) & 0.75 (frozen) \\
& Norm $\left(10^{-4}\right)$ & 5.0 (frozen) & 5.0 (frozen) \\
DISKBB & $T_{\text {in }}(\mathrm{keV})$ & 0.51 (frozen) & 0.51 (frozen) \\
& Norm & 78 (frozen) & 78 (frozen) \\
NTHCoMP & Gamma & 1.84 (frozen) & 1.84 (frozen) \\
& $k T_{\mathrm{e}}(\mathrm{keV})$ & 6 (frozen) & 6 (frozen) \\
& $k T_{\mathrm{bb}}(\mathrm{keV})$ & 0.75 (frozen) & 0.75 (frozen) \\
& inp_type $(0 / 1)$ & 0 (frozen) & 0 (frozen) \\
& Redshift & 0 (frozen) & 0 (frozen) \\
& Norm & 0.01 (frozen) & 0.01 (frozen) \\
\hline & $\chi^{2} /$ d.o.f. & $564.8 / 531$ & $554.8 / 541$ \\
\hline
\end{tabular}

Notes. The errors for each spectral parameter are quoted at $90 \%$ of statistical confidence.

consequence of this, we performed an individual analysis to the dip spectra of these two observations.

Since the dip is caused by a gradual covering of the central emitting area of the binary system, resulting in a progressive photoelectric absorption of the incoming flux, we used only the spectra extracted from the XIS data between 0.7 and $10 \mathrm{keV}$ where the dip is more evident.

According to the nature of the dipping phenomenon, we modeled the spectra of Obs 2 and Obs 3 using the warm_1seed model adopted for the continuum emission.

The spectral parameters that describe the continuum persistent emission, including the value of the equivalent hydrogen column of the phabs component related to the ISM, were kept fixed to those obtained using the warm_1seed model onto Spectrum 2; an exception to this was the component zxipcf. We also took into account that the warm absorber could also be responsible for a continuum optically thin Compton scattering, and for this reason we included a model of optically thin Compton scattering (cabs in Xspec), keeping the value of the hydrogen column of this component tied to that of the warm absorber. With this model the complex of features at about $6.7 \mathrm{keV}$ already detected in the persistent spectrum was not fitted; this resulted in a poor value of the $\chi$ (d.o.f.) of 787.44(534) and 1167.9(544) for Obs 2 and Obs 3, respectively. For this reason, we added a further zxipcf component, testing the possibility that the local partially ionized absorber showed a nonuniform value of the $\log (\xi)$ parameter. Hereafter this model is named 2warm_1seed model. The best-fit parameters obtained from the fits are reported in Table 3, while the best-fit model obtained for each observation is shown in Fig. 4 with the relative residuals.
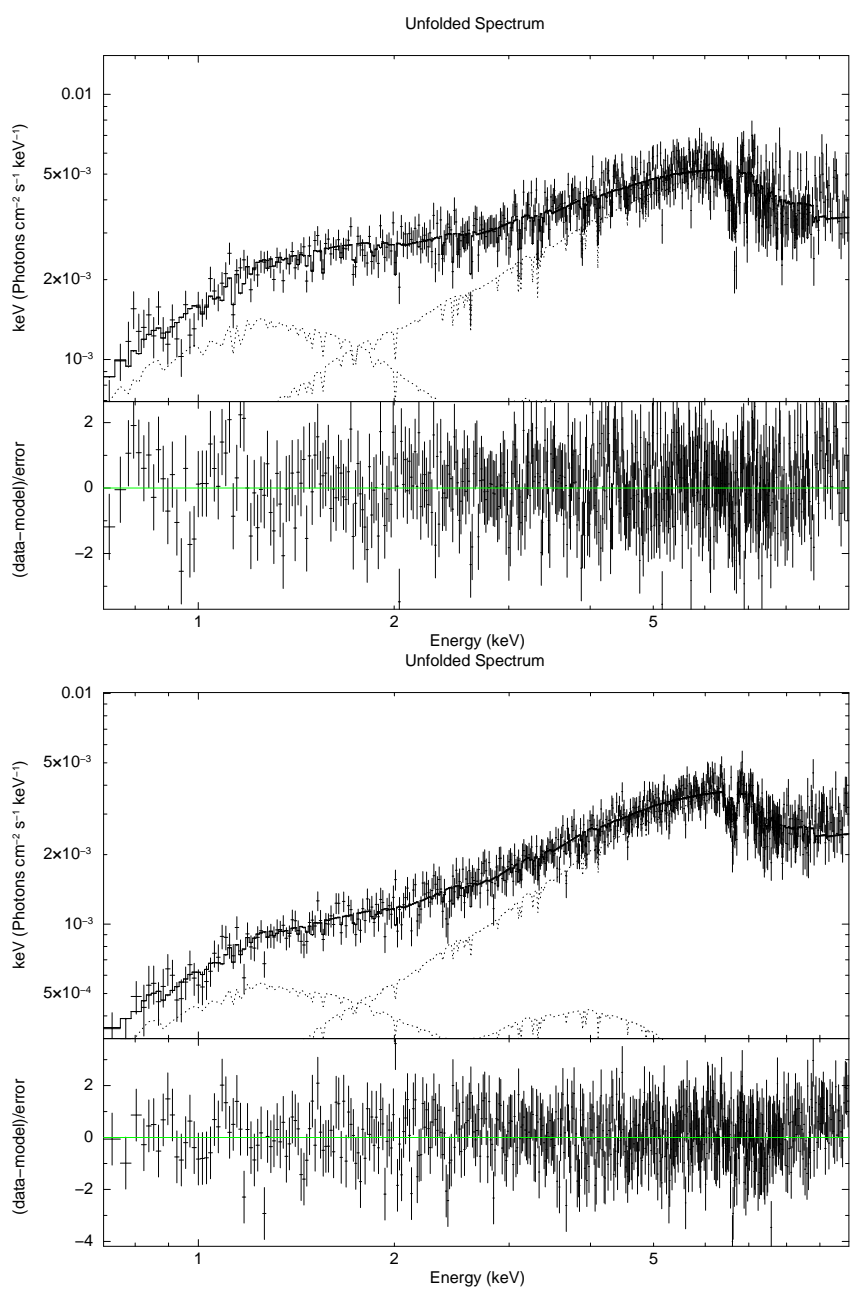

Fig. 4. Best-fit model for the dip spectra obtained from the Suzaku/XIS03 data of ObsID 409032010 (Obs2, upper plot) and ObsID 409032010 (Obs3, bottom plot). The residuals with respect to the adopted model are shown in units of sigma in the bottom panel of each plot.

The spectral parameters of Obs 1 and Obs 2 are quite similar and the components with the larger hydrogen columns $N_{\mathrm{H}}$ are characterized by the higher value of $\log (\xi)$ and smaller covering fraction. The most ionized component shows an average value of $\log (\xi)$ of about 3.2 in the two observations that well fits the residuals in absorption at about $6.7 \mathrm{keV}$. We obtain a $\chi^{2}$ (d.o.f.) of 564.8(531) and 554.8(541), for Obs 2 and Obs 3, respectively. This results in a significant statistical improvement of the fit with respect to the previous fit because the $F$-test probability that the improvements occur by chance is about $5 \times 10^{-38}$ and $5 \times 10^{-87}$, respectively.

\section{Discussion}

\subsection{Persistent spectrum}

We analyzed and modeled the continuum and dip spectra of the LMXB source XB 1916-053, using high statistics observations collected by the Suzaku space mission at different times.

Fitting the spectra of the persistent emission with the 1-seed model, we observed absorption features that suggested the presence of a local partially ionized absorber. For this reason, we adopted the warm_1seed model that includes the contribution of the warm partially ionized local absorber zxipcf. Fitting 
the warm_1seed model to the Spectrum 1, we obtained spectral parameters that are in good agreement with the results of Zhang et al. (2014).

The fit returns a value of the photon index of $\Gamma=2.1_{-0.2}^{+0.3}$ and a temperature of the Comptonizing cloud of $k T_{\mathrm{e}}=7 \pm 1 \mathrm{keV}$. Moreover, the normalization parameter of the diskbb component allowed us to estimate the apparent inner radius of the accretion disk as $R_{\mathrm{in}}=32 \pm 10 \mathrm{~km}$, conveniently corrected for the square of the color factor of Shimura \& Takahara (1995). This estimation assumed an inclination angle of $i=70 \pm 10 \mathrm{deg}$ (van Paradijs et al. 1988; Frank et al. 1987) and a distance of $D=9.3 \mathrm{kpc}$ as evaluated by Yoshida (1993) with an associated error of $20 \%$ (Zhang et al. 2014). Such value of the inner radius is in agreement with other radius measurements performed in other atoll sources (e.g., Di Salvo et al. 2015) and suggests that the accretion disk extends close to the NS surface, as observed by Zhang et al. (2014) who analyzed the same observation. The application of the same model to Spectrum 2 returns similar results. The values of the photon index and temperature of the electron cloud ( $\Gamma=1.84_{-0.05}^{+0.04}$ and $K T_{\mathrm{e}}=6 \pm 1 \mathrm{keV}$, respectively) are compatible with those obtained from the previous fit, suggesting that no evident changes in the corona have been observed in the two observations that are separated by a time span of eight years. In this case we infer an inner radius for the accretion disk of $41_{-13}^{+15} \mathrm{~km}$ that is in agreement with the previous estimation.

We extracted the unabsorbed bolometric flux between 0.1 and $100 \mathrm{keV}$ for each spectrum modeled with the warm_1seed model, obtaining $\Phi=(5.8 \pm 0.6) \times 10^{-10} \mathrm{erg} \mathrm{cm}^{-2} \mathrm{~s}^{-1}$ and $\Phi=(5.4 \pm 0.5) \times 10^{-10} \mathrm{erg} \mathrm{cm}^{-2} \mathrm{~s}^{-1}$ for Spectrum 1 and Spectrum 2, respectively. Assuming a distance to the source of $d=9.3 \pm 1.4 \mathrm{kpc}$ (Yoshida 1993), we obtained an unabsorbed bolometric luminosity in the $0.1-100 \mathrm{keV}$ energy band of $L=$ $(6 \pm 2) \times 10^{36} \mathrm{erg} \mathrm{s}^{-1}$ and $L=(5 \pm 2) \times 10^{36} \mathrm{erg} \mathrm{s}^{-1}$ for Spectrum 1 and Spectrum 2, respectively. This suggests that XB 1916-053 is a persistent source that does not show important changes of flux as also shown by the All-Sky Monitor (ASM) on board the Rossi X-ray Timing Explorer (RXTE) (Homer et al. 2001).

We also evaluated the optical depth of the Comptonizing electron cloud for Spectrum 1 and Spectrum 2, using the relation of Zdziarski et al. (1996)

$\Gamma=\left[\frac{9}{4}+\frac{1}{\tau\left(1+\frac{\tau}{3}\right)\left(\frac{k T_{\mathrm{e}}}{m_{\mathrm{e}} c^{2}}\right)}\right]^{1 / 2}-\frac{1}{2}$,

according to the spectral parameters obtained by fitting the warm_1seed model onto the data.

We obtained an optical depth of $\tau=6_{-2}^{+1}$ for Spectrum 1 and $\tau=8 \pm 1$ for Spectrum 2, respectively, indicating that the corona is optically thick.

An estimation of the radius of the region from which the seed photons are emitted, can be obtained using the relation of in 't Zand et al. (1999), assuming a spherical geometry of the corona,

$R_{0}=3 \times 10^{4} d\left(\frac{f_{\mathrm{bol}}}{1+y}\right)^{1 / 2}\left(k T_{\mathrm{bb}}\right)^{-2}$,

where $d$ is the distance to the source in kpc, $f_{\text {bol }}$ is the unabsorbed bolometric flux extrapolated from the Comptonization component in erg cm $\mathrm{cm}^{-2} \mathrm{~s}^{-1}, k T_{\mathrm{bb}}$ is the temperature of the seed photons in $\mathrm{keV}$, and $y=4 k T_{\mathrm{e}} \max \left[\tau, \tau^{2}\right] /\left(m_{\mathrm{e}} c^{2}\right)$ is the Compton parameter, in which $k T_{\mathrm{e}}$ is the electron temperature in $\mathrm{keV}$.
On the basis of the results we obtained fitting the warm_1seed model onto the data, we calculated a Comptonization parameter $y=0.6_{-0.4}^{+0.2}$ and $y=3 \pm 1$ for Spectrum 1 and Spectrum 2 and a radius for the seed photons of $R_{0}=10_{-3}^{+2} \mathrm{~km}$ and $R_{0}=14_{-3}^{+6} \mathrm{~km}$, respectively, already corrected for the color factor $\psi=1.7$ of Shimura \& Takahara (1995). This value agrees with the typical radii of NSs and suggests that the emission from the NS surface actually contributes to provide photons that are Compton-scattered by the corona. This result is also consistent with the quite low value of normalization found for the bbody component. The direct emission of the NS surface, indeed, could be totally intercepted by the optically thick corona, then contributing as source of seed photons.

The obtained optical depth and the values found for the temperature of the electron cloud seem to suggest that in the two spectra the physical conditions of the corona did not change and that the accretion disk maintains its inner radius close to the NS surface, according to what is usually observed for systems in a soft spectral state.

In their work, Zhang et al. (2014) suggested that the 2-seed model better describes the Comptonization process that contributes to the observed spectrum of the source. Furthermore, in Zhang et al. (2016) the persistent spectrum of the dipping and eclipsing LMXB source EXO 0748-676 is modeled with a 2-seed model on the basis of the fact that the authors obtained a radius of the seed photons that is larger than the radius of the NS, and then a contribution of photons is required for the Comptonization. This is not the case for our data, for which the adoption of the warm_1seed model implies that the photons that are Compton-scattered in the corona are emitted in a region with a radius that is compatible with the radius of the NS. However, to test if the contribution of the photons emitted by the disk is actually negligible, in Sect. 3 we decided to model our spectra with the warm_2seed model as well.

The results obtained with this model, reinforced by the $F$-test, suggested that a double Comptonization process is not needed on the basis of the current statistics of the data, while the fit seems to suggest an inner radius of the accretion disk that extends relatively close to the NS surface.

Adopting the warm_2seed model and using Eq. (1), we obtained an optical depth of $\tau=3_{-2}^{+8}$ and $\tau=8_{-3}^{+5}$ for Spectrum 1 and Spectrum 2, respectively. Using these optical depths and the electron temperature $k T_{\mathrm{e}}$ obtained from the fit (see Table 2) we found a Comptonization parameter $y=1_{-1}^{+5}$ and $y=3_{-2}^{+4}$, for Spectrum 1 and Spectrum 2, respectively.

In this case, using Eq. (2) we inferred that the radius of the region from which the seed photons are emitted is $R_{0}<3 \mathrm{~km}$ for Spectrum 1, while it is equal to $R_{0}=5_{-2}^{+6} \mathrm{~km}$ for Spectrum 2. The radius obtained for Spectrum 1 is fairly small, if compared to the typical value of the NS radius (i.e., $\sim 0 \mathrm{~km}$ ). This discrepancy could be caused by a low statistics of the Spectrum 1 or could alternatively suggest that only a fraction of the NS surface is responsible for the emission of seed photons that are Compton scattered. On the other hand, the higher statistics of Spectrum 2 allowed us to better constrain the value of $R_{0}$ that is in agreement, within the error, with the typical value of NSs radii.

Taking into account all these results, we are not able to break the degeneracy of the adopted models, even through the analysis of a spectrum with higher statistics. For this reason we take into account the results of the warm_1seed model, which not only returns a better value of the $\chi^{2}$ but is also less parameterized.

The presence of a local partially ionized absorbing material was justified by the observation of several absorption features in the spectra. Fitting Spectrum 1 and Spectrum 2 with the 
1-seed model we observed the absorption $\mathrm{K} \alpha$ lines of Fe xxv and Fe xxvi. These lines are quite strong and were detected with a level of statistical confidence of $4 \sigma$ and $11 \sigma$ for the Fe xxv and Fe xxvi $\mathrm{K} \alpha$ lines, respectively, in the case of Spectrum 1, and with a level of confidence of $9 \sigma$ and $17 \sigma$ for Spectrum 2. Iaria et al. (2006) reported that these features originate at the outer rim of the accretion disk, where the plasma is colder but also partially ionized. The strength of these lines, and in particular the difference in the line depth, can be explained as a difference of the number of the Fe xxv ions with respect to Fe xxvI, for the $\mathrm{K} \alpha$ transitions. To explain the larger depth of the Fe xxvI absorption line, we should expect a prevalence of these ions with respect to the Fe xxv ions. To estimate the difference of population for the two ions, we used the relation provided by Spitzer (1978), i.e.,

$\frac{W_{\lambda}}{\lambda}=\frac{\pi e^{2}}{m_{\mathrm{e}} c^{2}} N_{j} \lambda f_{i j}$,

where $N_{j}$ is the column density for the relevant species, $f_{i j}$ is the oscillator strength, $W_{\lambda}$ is the equivalent width of the line, and $\lambda$ is the wavelength expressed in centimeters.

The values of the oscillator strength are tabulated in Verner \& Yakovlev (1995) and are equal to $f_{i j}=0.798$ and $f_{i j}=0.277$ for the Fe xxv and Fe xxvI K $\alpha$ transitions, respectively. From the 1-seed model applied to Spectrum 2 we find an equivalent width of $W_{\lambda}=(5 \pm 2) \mathrm{eV}$ and $W_{\lambda}=(17 \pm 2) \mathrm{eV}$ for the Fe XXv and Fe XxvI $\mathrm{K} \alpha$ absorption lines, respectively. The errors associated with the equivalent widths are reported with a level of statistical confidence of $1 \sigma$. Then, using Eq. (3) we obtain $N_{\mathrm{Fe} 25, \mathrm{~K} \alpha}=(0.6 \pm 0.2) \times 10^{17} \mathrm{~cm}^{-2}$ and $N_{\mathrm{Fe} 26, \mathrm{~K} \alpha}=$ $(5.6 \pm 0.7) \times 10^{17} \mathrm{~cm}^{-2}$, whose ratio suggest a predominance of Fe Xxvi ions with respect to the Fe xxv by a factor of about 9, and this probably explains the difference in the depth of the two lines. These strong absorption lines were already noticed by Zhang et al. (2014), Boirin et al. (2004), and Iaria et al. (2006), where a full diagnostics of the absorption lines was performed using Chandra data.

The Spectrum 2, however, has a higher statistics with respect to the Spectrum 1 and in addition to the $\mathrm{K} \alpha$ absorption lines it also shows residuals that we found to be consistent with the $\mathrm{K} \beta$ absorption lines of Fe xxv and Fe xxvI, respectively.

Because of the moderate energy resolution of Suzaku, these two absorption lines have been modeled keeping their widths fixed to the same value of the $\mathrm{K} \alpha$ lines, i.e., $20 \mathrm{eV}$. The inclusion of these two lines in the 2-seed model actually improves the fit with respect to the case in which only the $\mathrm{K} \alpha$ lines were included because the probability for such improvement to occur by chance is $2 \times 10^{-3}$.

The presence of the Fexxv $\mathrm{K} \beta$ line has been suggested by Boirin et al. (2004) with XMM-Newton data, and is confirmed in this work taking advantage of the statistics of Spectrum 2. We detected this line with a level of confidence of $3 \sigma$ and about $4 \sigma$ using the 1-seed and 2-seed models, respectively. Moreover, we also detected a line consistent with a Fe XxvI $\mathrm{K} \beta$ absorption line with a level of confidence of about $4 \sigma$, independently from the adopted model. Actually, this line is located in the energy range in which Boirin et al. (2004) observed some weak residuals that assumed to be caused by the presence of Ni xxvII. We introduce the further possibility that this feature is due to the presence of Fe xxvi ions making $\mathrm{K} \beta$ transitions.

Taking into account Eq. (3), we can estimate the number population of the two species of ions. From the 1-seed model fitted to Spectrum 2 we obtain an equivalent width of $W_{\lambda}=(5 \pm 3) \mathrm{eV}$ for both the Fe Xxv and Fe xxvI K $\beta$ transitions, respectively, using the equivalent oscillator strengths $f_{i j}=0.156$ and $f_{i j}=0.079$, respectively, tabulated in Verner \& Yakovlev (1995). We obtain $N_{\mathrm{Fe} 25, \mathrm{~K} \beta}=(3 \pm 2) \times 10^{17} \mathrm{~cm}^{-2}$ and $N_{\mathrm{Fe} 26, \mathrm{~K} \beta}=(6 \pm 3) \times 10^{17} \mathrm{~cm}^{-2}$, with a ratio that suggests a predominance by a factor of about 2 of Fe xxvi ions making $\mathrm{K} \beta$ transitions with respect to Fe xxv ions making the same transition.

In addition to these discrete features, we also detected an absorption edge in Spectrum 2 at $0.87 \mathrm{keV}$. This edge is compatible with a $\mathrm{O}$ VIII $\mathrm{K}$ absorption edge that has already been observed in several LMXB systems (see, e.g., Iaria et al. 2016; Cottam et al. 2001). This feature is necessary to fit Spectrum 2 with all the adopted models. In particular, taking into account that the local warm absorbed in the system has a considerably high ionization state (as suggested by the $\log (\xi)$ value of about 5 ), it is probable that the $\mathrm{O}$ VIII ions that are responsible for this feature are generated in a relatively colder region.

\subsection{Dip spectrum}

The dip spectrum, on the other hand, was studied considering the XIS data of the ObsID 409032010 (Obs 2) and 409032020 (Obs 3 ). The resulting spectra have a net exposure of about $26 \mathrm{ks}$ and $46 \mathrm{ks}$ for Obs 2 and Obs 3, respectively.

The results of the fits performed using the 2warm_1seed model show that the introduction of a further warm absorber into the warm_1seed model is statistically needed by the available data, which for this reason seem to suggest that the absorber is not uniformly ionized. Moreover, even though the estimated covering fractions of the absorbers are similar passing from Obs 2 to Obs 3, on the other hand the absorber provided with the higher value of $\log (\xi)$ increases its equivalent hydrogen column density.

The local absorbing materials have an equivalent hydrogen column density of $N_{\mathrm{H}}=\left(15_{-3}^{+2}\right) \times 10^{22} \mathrm{~cm}^{-2}$ and $N_{\mathrm{H}}=(67 \pm 4) \times$ $10^{22} \mathrm{~cm}^{-2}$ in Obs 2 , or alternatively of $N_{\mathrm{H}}=(17 \pm 1) \times 10^{22} \mathrm{~cm}^{-2}$ and $N_{\mathrm{H}}=\left(91_{-4}^{+6}\right) \times 10^{22} \mathrm{~cm}^{-2}$ in Obs 3 . The fraction of the persistent spectrum that is covered by this material is on average equal to the $85 \%$ and $60 \%$ for the less and highly ionized absorbers, respectively, in both the observations, while the ionization parameters $\log (\xi)$ are on average equal to 0.87 and 3.14 in each observation. These results suggest that the bulge probably does not significantly change its morphology even though the ionization state and density of the matter could show variations in timescales on the order of few orbital periods.

A rough estimation of the distance of the warm absorber could be evaluated by means of the ionization parameter $\xi$, which determines the ionization state of the material of the warm absorber. According to Tarter et al. (1969), this parameter is defined as

$\xi=\frac{L}{n_{\mathrm{H}} r^{2}}$,

where $L$ is the unabsorbed luminosity of the illuminating source in erg s $\mathrm{s}^{-1}, n_{\mathrm{H}}$ is the total density of hydrogen in $\mathrm{cm}^{-3}$, and $r$ is the distance of the absorber from the center of the system in centimeters. Assuming that the radial size of the absorber $\Delta R$ is much lower that the typical value of the outer radius of the accretion disk, that is $r_{\text {disk }}=4 \times 10^{10} \mathrm{~cm}$ as reported in Iaria et al. (2006), we can infer upper limits on the distances of the local absorbers with respect to the position of the NS. We estimate that the less ionized absorber of Obs 2 is located at $r \leq 5 \times 10^{11} \mathrm{~cm}$, while the more ionized at $r \leq 1 \times 10^{10} \mathrm{~cm}$. In Obs 3 the less ionized absorber is located at $r \leq 3 \times 10^{11} \mathrm{~cm}$, while the more ionized is located at $r \leq 1 \times 10^{10} \mathrm{~cm}$. From these results we can notice that the upper limits on the distances inferred for the more ionized absorber in 
both observations are identical and actually explain the presence of the absorption features also observed into the persistent spectra, which usually form in the inner parts of the accretion disks. At the same time, these results rescale for the first time the distance of the absorber that Iaria et al. (2006) arbitrarily assumed to be located at the outer rim of the accretion disk.

At the same time, we are not able to constrain the distance of the weakly ionized absorbers of both observations. For this reason, according to their weak ionization parameters returned by the fits, we assumed we could locate these absorbers at the disk outer rim, trying to estimate their density $n_{\mathrm{H}}$ and thickness $\Delta R$. With this assumption, using the bolometric luminosity of the source in the range $0.1-100 \mathrm{keV}$ (i.e., $\left.L=(5 \pm 2) \times 10^{36} \mathrm{erg} \mathrm{s}^{-1}\right)$ and the values of $\xi$ we estimate that the total density of hydrogen in the weakly ionized absorber of Obs 2 is of about $n_{\mathrm{H}} \sim 7 \times$ $10^{14} \mathrm{~cm}^{-3}$, which implies a thickness of the absorber of $\Delta R \sim$ $2 \times 10^{8} \mathrm{~cm}$ (i.e., about the $0.5 \%$ of the accretion disk radius). On the other hand, for the weakly ionized absorber of Obs 3 we estimate a hydrogen density of $n_{\mathrm{H}} \sim 2 \times 10^{14} \mathrm{~cm}^{-3}$, which implies a thickness of $\Delta R \sim 8 \times 10^{8} \mathrm{~cm}$ (i.e., about the $2 \%$ of the disk radius).

\section{Conclusions}

In this work we report the results of a spectral analysis performed on both the persistent and the dip spectra of the LMXB system XB 1916-053 using Suzaku data. Even though we expect the photons emitted by the accretion disk to be partly scattered by the electron corona, the considerable statistics offered by the most recent observations is not sufficient to discriminate if the Comptonization of these photons is relevant with respect to that of the only photons emitted by the NS surface. We therefore preferred to adopt the single Comptonization description as the bestfit model because in that approach there is less parameterization of the data; however we took the contribution of the local partially ionized absorber into account.

The source has been found in a quite soft spectral state independent of the model adopted to fit the continuum spectrum. The achievement of a greater statistics, on the other hand, allowed us to detect various discrete absorption features, and in particular the $\mathrm{K} \beta$ absorption lines of Fe xxv and Fe xxvI, in addition to the $\mathrm{K} \alpha$ transition lines of the same ions, already reported in literature. The equivalent widths of these absorption lines seem to be consistent with a higher number of Fe Xxvi ions with respect to the Fe xxv. In addition, we detected a $\mathrm{O}$ VIII $\mathrm{K}$ absorption edge, which suggests the presence of this ion at large radial distances from the NS (possibly in the outer parts of the accretion disk).

The analysis of the dip spectrum highlighted how the dip itself could be explained as a gradual covering of the persistent spectrum by a cold and partially nonuniformly ionized bulk of matter; according to our results, this dip can be located at a distance from the NS that is lower than $1 \times 10^{10} \mathrm{~cm}$, that is at about $25 \%$ of the disk outer rim according to the existing estimations in literature. The weakly ionized part of the absorber, on the other hand, is characterized by a total density of hydrogen that slightly variates between $7 \times 10^{14} \mathrm{~cm}^{-3}$ and $2 \times 10^{14} \mathrm{~cm}^{-3}$ in the two observations and by a thickness of the absorber that variates between $8000 \mathrm{~km}$ and $4000 \mathrm{~km}$. In order to better constrain the persistent absorption features and their relative widths, we encourage high exposure observations to be performed by space missions, such as Athena, using high energy resolution.

Acknowledgements. This research has made use of data and/or software provided by the High Energy Astrophysics Science Archive Research Center (HEASARC), which is a service of the Astrophysics Science Division at NASA/GSFC and the High Energy Astrophysics Division of the Smithsonian Astrophysical Observatory. This research has made use of the VizieR catalogue access tool, CDS, Strasbourg, France. We acknowledge financial contribution from the agreement ASI-INAF I/037/12/0. We also acknowledge financial contribution from the agreement ASI-INAF n.2017-14-H.0. We acknowledge support from the HERMES Project, financed by the Italian Space Agency (ASI) Agreement n. 2016/13 U.O. Part of this work has been funded using resources from the research grant "iPeska" (P.I. Andrea Possenti) funded under the INAF national call Prin-SKA/CTA approved with the Presidential Decree 70/2016. S.M.M. thanks the research project "Stelle di neutroni come laboratorio di Fisica della Materia Ultra-densa: uno studio multifrequenza" financed by Regione Autonoma della Sardegna (scientific project manager prof. Luciano Burderi) in which part of this work was developed. The authors also acknowledge the HXD team for the useful help during the data analysis step.

\section{References}

Becker, R. H., Smith, B. W., Swank, J. H., et al. 1977, ApJ, 216, L101 Boirin, L., Parmar, A. N., Barret, D., Paltani, S., \& Grindlay, J. E. 2004, A\&A, 418, 1061

Boirin, L., Méndez, M., Díaz Trigo, M., Parmar, A. N., \& Kaastra, J. S. 2005, A\&A, 436, 195

Church, M. J., Dotani, T., BałuciŃska-Church, M., et al. 1997, ApJ, 491, 388

Cottam, J., Kahn, S. M., Brinkman, A. C., den Herder, J. W., \& Erd, C. 2001, A\&A, 365, L277

di Salvo, T., D’Aí, A., Iaria, R., et al. 2009, MNRAS, 398, 2022

Di Salvo, T., Iaria, R., Matranga, M., et al. 2015, MNRAS, 449, 2794

Frank, J., King, A. R., \& Lasota, J.-P. 1987, A\&A, 178, 137

Galloway, D. K., Muno, M. P., Hartman, J. M., Psaltis, D., \& Chakrabarty, D. 2008, ApJS, 179, 360

Grindlay, J. E., Bailyn, C. D., Cohn, H., et al. 1988, ApJ, 334, L25

Homer, L., Charles, P. A., Hakala, P., et al. 2001, MNRAS, 322, 827

Iaria, R., Di Salvo, T., Lavagetto, G., Robba, N. R., \& Burderi, L. 2006, ApJ, 647, 1341

Iaria, R., Di Salvo, T., Gambino, A. F., et al. 2015, A\&A, 582, A32

Iaria, R., Di Salvo, T., Del Santo, M., et al. 2016, A\&A, 596, A21

in 't Zand, J. J. M., Verbunt, F., Strohmayer, T. E., et al. 1999, A\&A, 345, 100

Koyama, K., Tsunemi, H., Dotani, T., et al. 2007, PASJ, 59, 23

Miller, L., Turner, T. J., Reeves, J. N., et al. 2007, A\&A, 463, 131

Mitsuda, K., Inoue, H., Koyama, K., et al. 1984, PASJ, 36, 741

Mitsuda, K., Bautz, M., Inoue, H., et al. 2007, PASJ, 59, S1

Nelemans, G., Jonker, P. G., \& Steeghs, D. 2006, MNRAS, 370, 255

Piraino, S., Santangelo, A., Di Salvo, T., et al. 2007, A\&A, 471, L17

Reeves, J., Done, C., Pounds, K., et al. 2008, MNRAS, 385, L108

Shimura, T., \& Takahara, F. 1995, ApJ, 445, 780

Smale, A. P., Mason, K. O., White, N. E., \& Gottwald, M. 1988, MNRAS, 232, 647

Spitzer, L. 1978, Physical Processes in the Interstellar Medium (New York: Wiley-Interscience)

Takahashi, T., Abe, K., Endo, M., et al. 2007, PASJ, 59, 35

Tarter, C. B., Tucker, W. H., \& Salpeter, E. E. 1969, ApJ, 156, 943

van Paradijs, J., van der Klis, M., \& Pedersen, H. 1988, A\&AS, 76, 185

Verner, D. A., \& Yakovlev, D. G. 1995, A\&AS, 109, 125

Verner, D. A., Ferland, G. J., Korista, K. T., \& Yakovlev, D. G. 1996, ApJ, 465, 487

Wilms, J., Nowak, M. A., Boyd, P., et al. 2000, AAS/High Energy Astrophysics Division \#5, Bull. Am. Astron. Soc., 32, 1247

Yoshida, K. 1993, PhD thesis, Tokyo University, Japan

Zdziarski, A. A., Johnson, W. N., \& Magdziarz, P. 1996, MNRAS, 283, 193

Zhang, Z., Makishima, K., Sakurai, S., Sasano, M., \& Ono, K. 2014, PASJ, 66, 120

Zhang, Z., Sakurai, S., Makishima, K., et al. 2016, ApJ, 823, 131

Życki, P. T., Done, C., \& Smith, D. A. 1999, MNRAS, 309, 561 